\title{
Robustness Evaluation of a Novel Proton Beam Geometry for Head and Neck Patients Treated with Pencil Beam Scanning Therapy
}

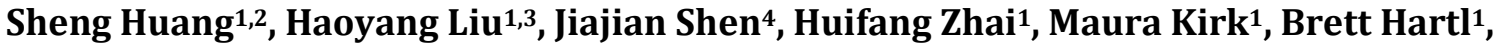 \\ Alexander Lin', James McDonough1, Stefan Both', Haibo Lin ${ }^{1,2 *}$ \\ ${ }^{1}$ Department of Radiation Oncology, University of Pennsylvania, Philadelphia, PA, USA \\ ${ }^{2}$ New York Proton Center, New York, NY, USA \\ ${ }^{3}$ New Jersey Proton Therapy Center, Somerset, NJ, USA \\ ${ }^{4}$ Mayo Clinic, Phoenix, AZ, USA \\ ${ }^{5}$ University Medical Center Groningen, Groningen, The Netherlands \\ Email: ^hlin@nyproton.com
}

How to cite this paper: Huang, S., Liu, H.Y., Shen, J.J., Zhai, H.F., Kirk, M., Hartl, B., Lin, A., McDonough, J., Both, S. and Lin, H.B. (2018) Robustness Evaluation of a Novel Proton Beam Geometry for Head and Neck Patients Treated with Pencil Beam Scanning Therapy. International Journal of Medical Physics, Clinical Engineering and Radiation Oncology, 7, 308-322. https://doi.org/10.4236/ijmpcero.2018.73025

Received: May 11, 2018

Accepted: July 15, 2018

Published: July 18, 2018

Copyright ( 92018 by authors and Scientific Research Publishing Inc. This work is licensed under the Creative Commons Attribution International License (CC BY 4.0).

http://creativecommons.org/licenses/by/4.0/

\section{Open Access}

\begin{abstract}
Background: To evaluate the robustness of head and neck treatment using proton pencil beam scanning (PBS) technique with respect to range uncertainty (RU) and setup errors (SE), and to establish a robust PBS planning strategy for future treatment. Methods and Materials: Ten consecutive patients were planned with a novel proton field geometry (combination of two posterior oblique fields and one anterior field with gradient dose match) using single-field uniform dose (SFUD) planning technique and the proton plans were dosimetrically compared to two coplanar arc VMAT plans. Robustness of the plans, with respect to range uncertainties ( $\mathrm{RU}= \pm 3 \%$ for proton) and setup errors $(\mathrm{SE}=2.25 \mathrm{~mm}$ for proton and VMAT), in terms of deviations to target coverage (CTV D98\%) and OAR doses (max/mean), were evaluated and compared for each patient under worst case scenarios. Results: Dosimetrically, PBS plans provided better sparing to larynx $(\mathrm{p}=0.005)$, oral cavity $(\mathrm{p}<0.001)$ and contralateral parotid $(\mathrm{p}=0.004)$ when compared to VMAT. CTV D98\% variations were higher from SE than from RU for proton plans $(-1.1 \% \pm 1.3 \%$ vs $-0.4 \%$ $\pm 0.7 \%$ for nodal CTV and $-1.4 \% \pm 1.2$ vs $-0.4 \% \pm 0.5 \% \%$ for boost CTV). Overall, the magnitudes of variation of CTV D98\% to combined SE and RU were found to be similar to the impact of the SE on the VMAT plans $(-1.6 \% \pm$ $1.9 \%$ vs $-1.7 \% \pm 1.4 \%$ for nodal CTV and $-1.9 \% \pm 1.6 \%$ vs $-1.3 \% \pm 1.5 \%$ for boost CTV). Compared to VMAT, a larger range of relative dose deviations were found for OARs in proton plans, but safe doses were maintained for cord $(41.8 \pm 3.6$ Gy for PBS and $41.7 \pm 3.9$ Gy for VMAT) and brainstem $(35.2 \pm$ 8.4 Gy for PBS and 36.2 \pm 5.1 Gy for VMAT) in worst case scenarios. Conclu-
\end{abstract}


sions: Compared to VMAT, proton plans containing three SFUD fields with superior-inferior gradient dose matching had improved sparing to larynx, contralateral parotid and oral cavity, while providing similar robustness of target coverage. Evaluation of OAR dose robustness showed higher sensitivities to uncertainties for proton plans, but safe dose levels were maintained for cord and brainstem.

\section{Keywords}

Head and Neck, Robustness, Proton Therapy, Pencil Beam Scanning

\section{Introduction}

The main goal of modern radiotherapy is to optimize the delivery of radiation dose such that the target is fully sterilized while maximally sparing the surrounding healthy tissue and organs. Especially in head and neck (HN) cancers, this goal is challenging to achieve because organs at risk (OAR) are generally proximal to target volumes. Huguenin et al. had a quality of life study for $\mathrm{HN}$ patients received radiotherapy and revealed the highest morbidity was probably the result of using large radiation fields [1]. Therefore, reduction in irradiated volume and dose to local organs may lower the risk and severity of radiation induced complications [2]. Advanced conventional delivery techniques such as intensity modulated radiation therapy (IMRT) and volumetric modulated arc therapy (VMAT) have improved the outlook for OAR for $\mathrm{HN}$ patients in the past several decades [3]. Proton therapy has the potential to further improve OAR sparing and reduce integral dose resulting from the intrinsic physics of the proton beam [4].

Proton pencil beam scanning (PBS) technique is the current state of art of modern proton therapy. Generally, PBS dose can be shaped using either single-field uniform dose (SFUD) or intensity modulated proton therapy (IMPT) planning techniques. In SFUD, often called single-field optimization, fields are optimized simultaneously so that each field uniformly covers the whole target. In IMPT, fields are also optimized simultaneously, but each field does not uniformly cover the whole target and only the sum of all fields provides adequate and homogeneous target coverage, similar to IMRT. Although the flexibility of IMPT allows for creation of more complex dose distributions than SFUD, which improves OAR sparing in practice, concerns such as high sensitivity to uncertainties and lack of robustness for IMPT still exist [5]. In contrast, SFUD plans are considered to be more robust than IMPT due to less dose modulation and lower complexity of dose distributions for each individual field [6].

Uncertainties in proton therapy are expected from several sources. Setup errors (SE), considered only as shifts in conventional therapy, also cause dose distortions in proton therapy resulting from the displacement of heterogeneity in the beam path, to which protons are more sensitive than photons. Additionally, 
range uncertainties (RU) arise from conversion of computed tomography (CT) Hounsfield units (HU) to proton stopping power, CT artifacts, fluctuation of proton energy, anatomy change and so on. Typically, RU from $\pm 3 \%$ to $\pm 3.5 \%$ of $\mathrm{HU}$ is taken into consideration for routine clinical practice [7]. Uncertainties related to motion should be considered as well when evaluating proton therapy plans, especially for PBS treatments due to interplay effect [8]. As all of these uncertainties may result in significant deviations between planned and delivered dose distributions [9], it is very important to evaluate the robustness of proton therapy plans, especially for newly developed clinical protocols.

Several previous studies have investigated the robustness of proton therapy for HN cancer compared to photon techniques. Barten et al. studied OAR sparing and plan robustness for PBS proton planning techniques including SFUD and IMPT with more fields and compared these with VMAT photon plans. They reached conclusion that IMPT plans has improved OAR sparing but were less robust than SFUD and VMAT and robustness of the MFO plans did not increase with more fields [6]. Van der Voort et al. derived a "robustness recipe" for minimax robust optimization of IMPT treatment plans to ensure adequate CTV coverage for $\mathrm{HN}$ cancer patient relative to systematic setup, random setup and range errors [10]. Malyapa et al. evaluated the robustness of IMPT plans to setup and range uncertainties for $\mathrm{HN}$ patient and created a robustness database, which allows the identification of future plans that may benefit from alternative planning approaches to improve robustness [7]. Van de Water et al. quantified the impact of the degree of robustness against setup and range errors on OAR dose and normal tissue complication probabilities in IMPT proton therapy for HN cancer patient and concluded that minimizing setup errors should be given a higher priority than minimizing range errors [11].

In this work, a novel HN proton treatment planning geometry containing three SFUD fields (two posterior (oblique) fields and one anterior field, inversed "Y" shape) using superior-inferior gradient dose matching is presented. The dosimetrical advantages and plan robustness of this approach are first time evaluated relative to two-Arc VMAT plans, in terms of target and OAR dose levels.

\section{Materials and Methods}

\subsection{Patient Group, Simulation and Contouring}

Ten consecutive post-operative oropharynx HN patients treated at our center were studied. Clinical target volume (CTV) and OAR were delineated based on our departmental clinical protocol. Planning target volume (PTV) was created from CTV with $3 \mathrm{~mm}$ uniform expansion (Figures 1(a)-(c)) and PBS target volume used for proton dose optimization was generated from CTV with $5 \mathrm{~mm}$ uniform expansion. Treatments were planned with simultaneous integrated boost (SIB) technique to deliver 54 Gy to nodal PTV (PTV54)/CTV (CTV54) and 60 Gy to boost PTV (PTV60)/CTV (CTV60) in 30 fractions of $1.8 \mathrm{~Gy}$ and 2 Gy $(\mathrm{RBE}=1.1)$ respectively. 

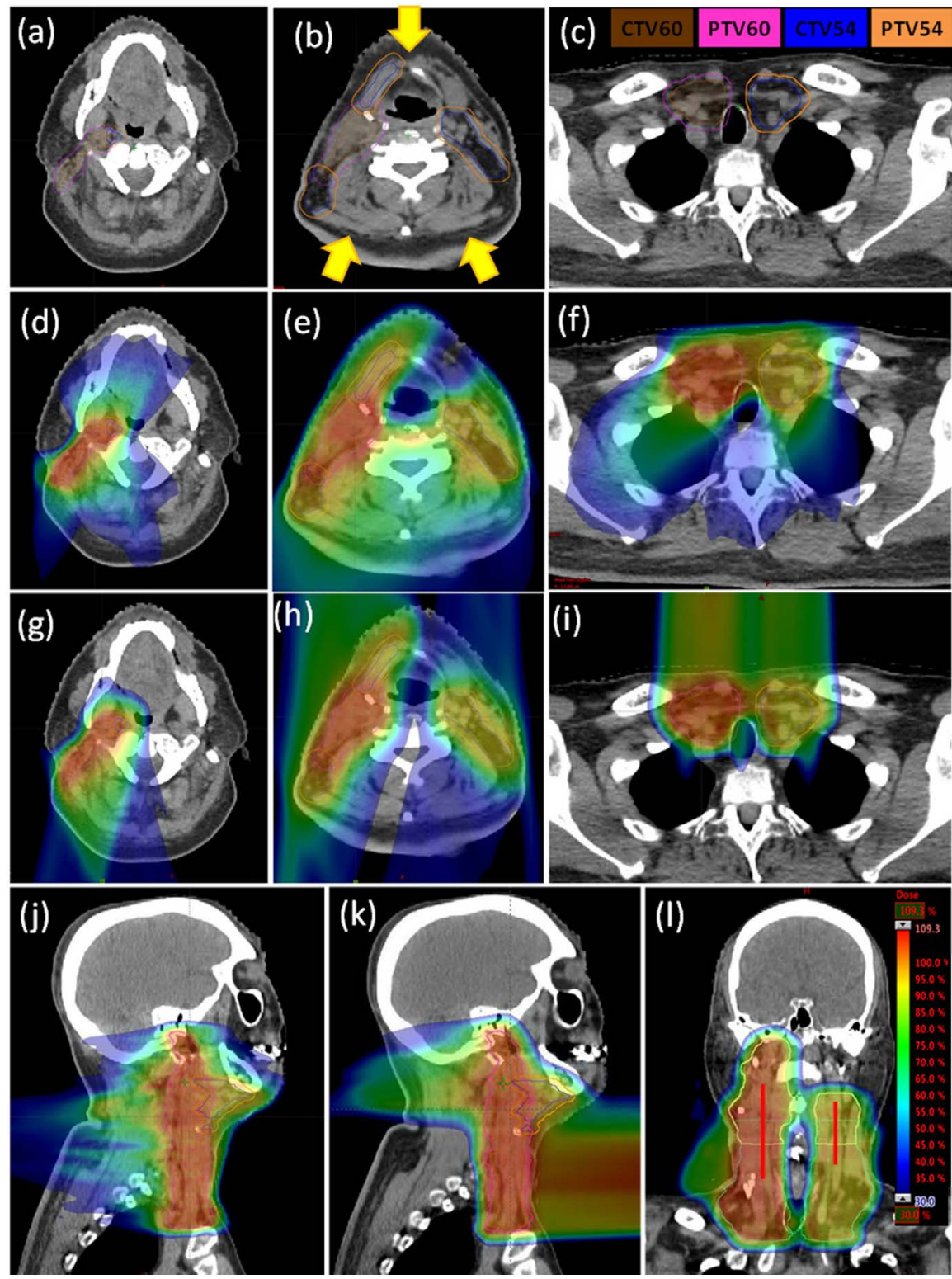

Figure 1. (a)-(c) Target volumes: PTV and CTV for HN planning of three axial cuts; three proton beams are indicated in (b) as 0, 195 and 160 degree. (d)-(f) Dose colorwash (30\% dose threshold for all) image of VMAT plan of three axial cuts. (g)-(i) Dose colorwash image of proton plan of three axial cuts: upper targets with two posterior fields ( $g$ ), lower targets with anterior field (i) and junction area with all three fields (h). (j) Dose colorwash of sagittal view of VMAT plan. (k) Dose colorwash of sagittal view of proton plan. (l) Dose colorwash with PBSTV overlapped at junction area; dose profiles indicated by the red lines are presented in Figure 2.

\subsection{Treatment Planning Techniques}

VMAT (Figures 1(d)-(f)) treatments were optimized with two full arcs using 6 MV photons in Eclipse (Varian Medical System, Palo Alto, CA) treatment planning system (TPS). Dose calculation was performed using the anisotropic analytical algorithm (Eclipse AAA v11.030) with grid size of $2.5 \mathrm{~mm}$.

Proton plans were created in Eclipse TPS using the proton convolution 


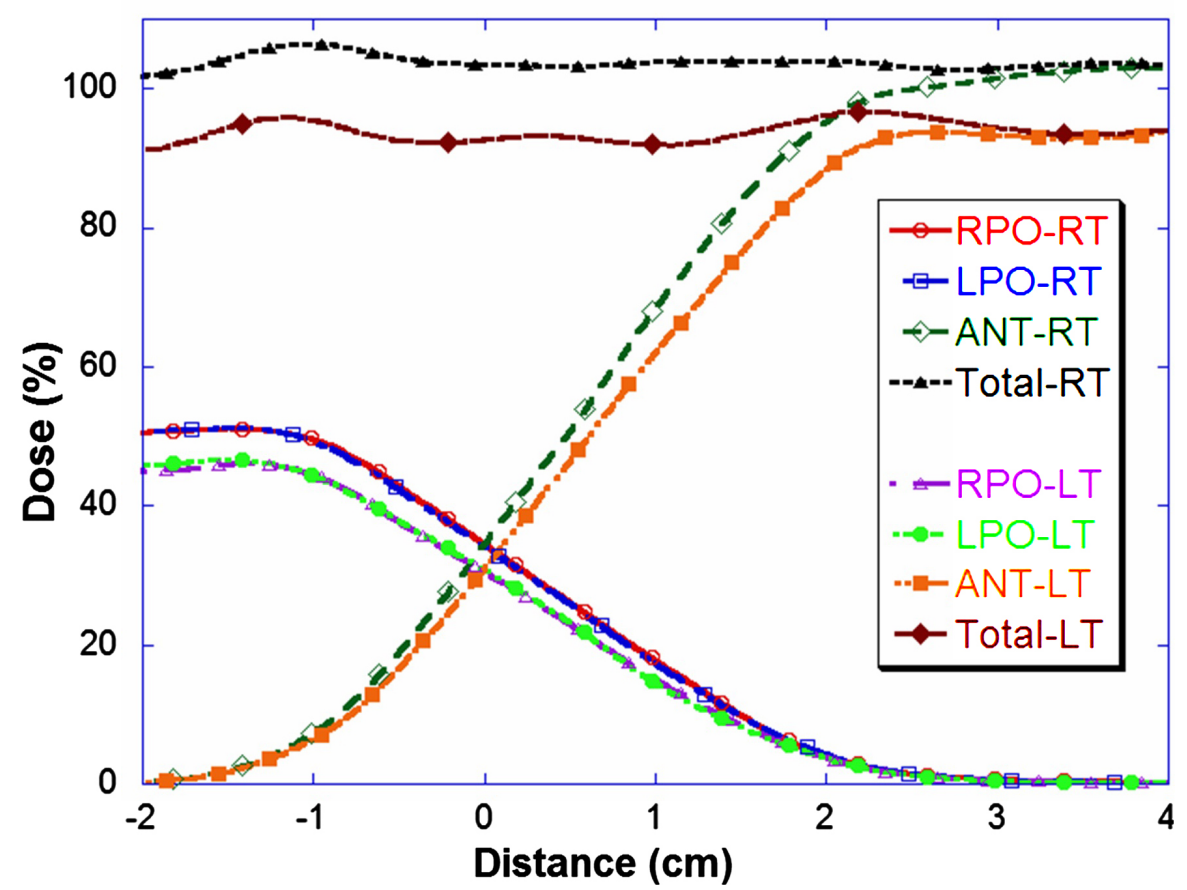

Figure 2. Dose profiles for individual field and the sum of all fields at the junction area indicated in Figure 1(1). Two dose levels of $60 \mathrm{~Gy}$ and $54 \mathrm{~Gy}$ are prescribed to PTV60 on patient's right (RT) side and PTV 54 on patient's left (LT) side. RPO-RT: right posterior oblique field at PTV60 junction area; LPO-RT: left posterior oblique field at PTV60 junction area; ANT-RT: anterior field at PTV60 junction area; Total-RT: dose sum from all three fields at PTV60 junction area. RPO-LT: right posterior oblique field at PTV54 junction area; LPO-LT: left posterior oblique field at PTV54 junction area; ANT-LT: anterior field at PTV54 junction area; Total-LT: dose sum from all three fields at PTV54 junction area.

superposition (Eclipse PCS 10.0.28) algorithm and simultaneous spot optimization with dose calculation grids of $2.5 \mathrm{~mm}$ and spot spacing of $5 \mathrm{~mm}$. Gradient dose optimization was used to match two sets of fields in craniocaudal direction, a method successfully applied to PBS craniospinal irradiation (CSI) [12]. All target volumes were separated into two sets along thyroid notch level with an overlap of $\sim 4 \mathrm{~cm}$ purposely. A subset of equally-spaced optimization structures was created in the overlap region and used to achieve smooth dose gradients in the craniocaudal direction for both upper and lower fields in this junction area (with the direction of the gradients opposed). As indicated in Figure 1(b), the posterior field set includes two posterior (oblique) fields with field angles ranging of $180^{\circ} \pm 20^{\circ}$ (Figure $1(\mathrm{~g})$ ) to treat the cranial targets at the primary site and upper and mid neck to spare the oral cavity. To avoid treating through dental, a single anterior field (Figure 1(i)) is added to cover the lower neck target volume. And then the sum of the plans was assessed for target coverage and OAR dose constraints, as well as the dose homogeneity, especially in the junction area (Figure 1(1)). SFUD was selected to plan each field set. But different to CSI treatment, $\mathrm{HN}$ target volume for each field set can typically fit in our maximal proton field $(34 \mathrm{~cm})$, thus all fields for HN planning share the same iso-center and do not 
need a couch shift between fields during treatment. This removes the potential setup errors through couch shifts during treatment.

Our proton system has minimal deliverable energy of $100 \mathrm{MeV}$, which translates to a range of $7.7 \mathrm{~cm} \mathrm{WET}$. To cover the shallow portion of targets, a thick couch $($ WET $7.35 \mathrm{~cm}$ ) and an anterior bolus (WET 7.16 cm) served as an alternative solution to machine range shifter for posterior and anterior fields respectively [13], so that sufficient coverage to the shallow portion of the target could be maintained. Online patient positioning was completed via alignment of bony anatomy around target volumes using two orthogonal kilo-voltage $(\mathrm{kV})$ based imaging guidance. The anterior bolus is placed in position after imaging-guided patient alignment. The vertical position of the bolus is indexed relative to couch surface to maintain a reproducible air gap between bolus and patient. As large air gaps could broaden the proton spot size [14], air gaps were kept as small as possible. Typically, the achieved air gap is $10 \mathrm{~cm}$ or below.

\subsection{Robustness Evaluation}

Worst case scenarios for SE were simulated for both proton and VMAT plans with simultaneous shifts in all three directions (8 combinations) similar to the method used in the study by Barten et al. [6]. As all patients were immobilized using a thermoplastic mask, a shift of $2.25 \mathrm{~mm}$ [7], which satisfied an $85 \%$ confidence level [15], was applied in three directions. The influence of RU was also investigated by introducing $\pm 3 \%$ errors on the $\mathrm{HU}$ values in proton planning CT ( 2 combinations) and was considered as negligible in VMAT planning. SE and RU were also combined (SREs: 16 combinations) for proton plans to evaluate the plan robustness in the worst-case scenario for both sources of uncertainty combined. Loss of target coverage and deviations to OAR doses were reported. For any given patient, multiple instances of overdose to critical organs, such as cord and brainstem, relative to their dose constraints, or a loss of CTV coverage $>5 \%$ triggered re-evaluation and potential re-optimization of the plan.

\section{Results}

\subsection{Dosimetric Evaluation and Comparison}

Table 1 summarizes the dosimetric results of proton and VMAT plans for 10 patients. A two tailed student $\mathrm{t}$ test was used to evaluate the statistical significance of dosimetric parameters from all plans. The planning objective for CTV coverage (D98\% $\geq 99 \%$ ) was achieved for all proton and photon plans, while the goal for PTV (D95\% $\geq 100 \%)$ coverage was only reached for 7 proton and 3 photon patients. The maximum deviation from the objective for PTV coverage was found to be $-1.2 \%$ of the prescription dose for one VMAT case, and all the others deviated $<1 \%$, which was considered clinically acceptable. The aim of less than $0.03 \mathrm{cc}$ of total body volume receiving $66 \mathrm{~Gy}$ or more was maintained in all plans. As shown in Figure 2, smooth dose gradient was achieved in the junction area (red lines indicated in Figure 1(1)) between matched proton fields for 
Table 1. Dosimetric parameters in Gy for targets and OARs in 10 patients planned with proton PBS and VMAT. Mean and standard deviation value of 10-patient results and the p-value based on a two tailed student $t$ test are also listed.

\begin{tabular}{|c|c|c|c|c|c|c|c|c|c|c|c|c|c|}
\hline VOI/patient & & Pt1 & Pt2 & Pt3 & Pt4 & Pt5 & Pt6 & Pt7 & Pt8 & Pt9 & Pt10 & Mean $\pm \Delta$ & $P$ value \\
\hline \multirow{2}{*}{$\begin{array}{l}\text { PTV54 } \\
\text { (D95\%) }\end{array}$} & PBS & 55.3 & 54.3 & 54.6 & 54.6 & 54.6 & 54.5 & 54.1 & 54.0 & 54.2 & 54.0 & $54.4 \pm 0.4$ & \multirow{2}{*}{0.08} \\
\hline & VMAT & 53.9 & 53.7 & 54.6 & 54.0 & 54.2 & 53.5 & 54.0 & 54.0 & 54.3 & 54.7 & $54.1 \pm 0.4$ & \\
\hline \multirow{2}{*}{$\begin{array}{l}\text { PTV60 } \\
\text { (D95\%) }\end{array}$} & PBS & 60.7 & 60.1 & 60.2 & 60.3 & 60.2 & 59.6 & 59.7 & 60.3 & 60.2 & 60.1 & $60.1 \pm 0.3$ & \multirow{2}{*}{0.07} \\
\hline & VMAT & 59.9 & 59.1 & 60.2 & 60.2 & 60.1 & 59.5 & 60.0 & 60.0 & 60.0 & 59.7 & $59.9 \pm 0.3$ & \\
\hline \multirow{2}{*}{$\begin{array}{l}\text { CTV54 } \\
\text { (D98\%) }\end{array}$} & PBS & 55.0 & 53.9 & 54.4 & 54.9 & 54.5 & 54.2 & 54.1 & 53.9 & 54.1 & 54.2 & $54.3 \pm 0.4$ & \multirow{2}{*}{0.02} \\
\hline & VMAT & 54.4 & 55.2 & 54.7 & 54.9 & 54.8 & 54.3 & 54.0 & 55.0 & 55.5 & 55.8 & $54.9 \pm 0.6$ & \\
\hline \multirow{2}{*}{$\begin{array}{l}\text { CTV60 } \\
\text { (D98\%) }\end{array}$} & PBS & 60.7 & 60.2 & 60.1 & 60.7 & 60.6 & 60.1 & 59.9 & 60.3 & 60.3 & 60.4 & $60.3 \pm 0.3$ & \multirow{2}{*}{0.11} \\
\hline & VMAT & 60.4 & 60.6 & 60.2 & 60.8 & 60.9 & 60.1 & 60.0 & 61.1 & 61.0 & 60.7 & $60.6 \pm 0.4$ & \\
\hline \multirow{2}{*}{$\begin{array}{l}\text { Brainstem } \\
\text { (D.max) }\end{array}$} & PBS & 33.7 & 30.5 & 37.0 & 42.3 & 31.3 & 47.4 & 42.9 & 38.0 & 23.5 & 30.8 & $35.7 \pm 7.2$ & \multirow{2}{*}{0.92} \\
\hline & VMAT & 39.4 & 38.1 & 38.8 & 33.1 & 25.9 & 37.0 & 33.7 & 33.8 & 35.7 & 44.6 & $36.0 \pm 4.9$ & \\
\hline \multirow{2}{*}{$\begin{array}{c}\text { Cord } \\
\text { (D.max) }\end{array}$} & PBS & 44.8 & 41.7 & 43.8 & 41.6 & 41.0 & 43.7 & 41.2 & 36.2 & 35.8 & 32.8 & $40.3 \pm 4.0$ & \multirow{2}{*}{0.78} \\
\hline & VMAT & 42.6 & 42.7 & 36.5 & 37.7 & 44.7 & 37.2 & 34.5 & 36.6 & 42.0 & 43.8 & $39.8 \pm 3.7$ & \\
\hline \multirow{2}{*}{$\begin{array}{l}\text { Oral cavity } \\
\text { (D.mean) }\end{array}$} & PBS & 4.2 & 2.1 & 6.9 & 5.9 & 1.3 & 7.4 & 2.0 & 3.2 & 1.6 & 2.1 & $3.7 \pm 2.3$ & \multirow{2}{*}{$<0.001$} \\
\hline & VMAT & 19.9 & 15.6 & 16.3 & 23.1 & 16.6 & 24.1 & 16.0 & 20.1 & 17.7 & 16.5 & $18.6 \pm 3.1$ & \\
\hline \multirow{2}{*}{$\begin{array}{l}\text { Contralateral submandibular } \\
\text { (D.mean) }\end{array}$} & PBS & 29.5 & 43.2 & 24.5 & 30.9 & 33.8 & 36.0 & 29.4 & 36.8 & 39.8 & 29.5 & $33.3 \pm 5.7$ & \multirow{2}{*}{0.71} \\
\hline & VMAT & 29.0 & 36.4 & 20.3 & 30.1 & 32.4 & 51.2 & 29.1 & 29.3 & 30.6 & 33.6 & $32.2 \pm 7.9$ & \\
\hline \multirow{2}{*}{$\begin{array}{l}\text { Contralateral Parotid } \\
\text { (D.mean) }\end{array}$} & PBS & 16.2 & 17.9 & 12.2 & 13.0 & 15.4 & 19.1 & 16.3 & 12.8 & 20.9 & 9.6 & $15.4 \pm 3.5$ & \multirow{2}{*}{0.004} \\
\hline & VMAT & 19.7 & 18.1 & 25.0 & 17.7 & 18.7 & 22.4 & 19.5 & 15.3 & 20.3 & 17.9 & $19.5 \pm 2.7$ & \\
\hline \multirow{2}{*}{$\begin{array}{l}\text { Ipsilateral Parotid } \\
\text { (D.mean) }\end{array}$} & PBS & 20.4 & 27.8 & 27.8 & 25.6 & 23.7 & 31.1 & 24.1 & 28.2 & 39.1 & 20.4 & $26.8 \pm 5.5$ & \multirow{2}{*}{0.56} \\
\hline & VMAT & 26.1 & 25.7 & 37.0 & 26.1 & 25.4 & 22.6 & 22.7 & 32.8 & 26.9 & 22.3 & $26.8 \pm 4.7$ & \\
\hline \multirow{2}{*}{$\begin{array}{l}\text { Larynx } \\
\text { (D.mean) }\end{array}$} & PBS & 29.0 & 31.1 & 27.3 & 27.1 & 25.5 & 31.3 & 27.3 & 24.5 & 23.0 & 25.5 & $27.2 \pm 2.7$ & \multirow{2}{*}{0.005} \\
\hline & VMAT & 32.4 & 33.7 & 38.1 & 28.9 & 33.3 & 27.3 & 34.6 & 27.1 & 34.7 & 28.1 & $31.7 \pm 3.9$ & \\
\hline
\end{tabular}

Note: VOI: volume of interest; D.max: maximal dose; D.mean: mean dose; Pt: patient; $\Delta$ : standard deviation.

proton plan. Clinically acceptable maximum dose constraints to cord and brainstem were also achieved for all plans.

In terms of OAR sparing, proton plans significantly improved the mean dose to oral cavity $(\mathrm{p}<0.001)$, larynx $(\mathrm{p}=0.005)$ and contralateral parotid $(\mathrm{p}=0.004)$ compared to VMAT plans. Sparing of OARs such as cord, brainstem, contralateral submandibular and ipsilateral parotid was patient specific and mostly relied on the relative location between organs and target volumes, as well as their shape along the beam direction and the level of overlaps between structures.

\subsection{Robustness Evaluation}

Table 2 summarizes the mean, standard deviation, and range of dose variations relative to the original plan found in the robustness study considering RU, SE, 
Table 2. Mean, standard deviation, minimum and maximum of dose variations in uncertainty comparison (between original plan and plan with SEs, RU and SREs) in $10 \mathrm{HN}$ patients for proton and VMAT plans.

\begin{tabular}{|c|c|c|c|c|c|c|c|c|}
\hline \multirow{3}{*}{ VOIs/Dose Metrics } & \multicolumn{4}{|c|}{$\Delta$ Mean $\pm \Delta$ Standard Deviation (\%) } & \multicolumn{4}{|c|}{$\Delta \operatorname{Max}(\%)$} \\
\hline & \multicolumn{2}{|c|}{ PBS } & \multicolumn{2}{|c|}{ VMAT } & \multicolumn{2}{|c|}{ PBS } & \multicolumn{2}{|c|}{ VMAT } \\
\hline & RU & SE & SREs & SE & RU & SE & SREs & SE \\
\hline PTV54 (D95\%) & $-0.7 \pm 0.9$ & $-1.8 \pm 1.0$ & $-2.5 \pm 1.6$ & $-4.4 \pm 1.3$ & -3.6 & -4.8 & -8.0 & -7.0 \\
\hline PTV60 (D95\%) & $-0.9 \pm 0.6$ & $-2.1 \pm 1.0$ & $-2.9 \pm 1.7$ & $-3.5 \pm 1.9$ & -2.0 & -5.8 & -9.1 & -7.5 \\
\hline CTV54 (D98\%) & $-0.4 \pm 0.7$ & $-1.1 \pm 1.3$ & $-1.6 \pm 1.9$ & $-1.7 \pm 1.4$ & -2.9 & -5.6 & -7.1 & -6.2 \\
\hline CTV60 (D98\%) & $-0.4 \pm 0.5$ & $-1.4 \pm 1.2$ & $-1.9 \pm 1.6$ & $-1.3 \pm 1.5$ & -1.9 & -6.2 & -7.3 & -6.2 \\
\hline Brainstem (D.max) & $0.3 \pm 2.7$ & $0.6 \pm 9.6$ & $1.0 \pm 10.0$ & $0.3 \pm 3.6$ & 4.1 & 17.5 & 20.6 & 6.3 \\
\hline Cord (D.max) & $0.1 \pm 1.0$ & $2.2 \pm 2.2$ & $2.2 \pm 2.6$ & $3.2 \pm 2.5$ & 1.3 & 13.2 & 13.5 & 8.1 \\
\hline Oral cavity (D.max) & $0.3 \pm 2.2$ & $0.2 \pm 0.8$ & $0.4 \pm 2.2$ & $0.1 \pm 3.3$ & 3.9 & 1.8 & 5.6 & 4.6 \\
\hline Submandibular (D.mean) & $0.2 \pm 13.3$ & $0.1 \pm 5.0$ & $-0.1 \pm 13.0$ & $1.0 \pm 7.3$ & 18.5 & 10.8 & 25.5 & 12.0 \\
\hline Contralatral Parotid (D.mean) & $0.1 \pm 3.7$ & $-0.2 \pm 5.4$ & $-0.1 \pm 6.2$ & $0.5 \pm 4.2$ & 5.0 & 10.0 & 15.0 & 6.6 \\
\hline Ipsilateral Parotid (D.mean) & $0.4 \pm 5.3$ & $0.4 \pm 8.1$ & $0.6 \pm 9.0$ & $0.8 \pm 6.7$ & 7.0 & 11.7 & 16.9 & 13.4 \\
\hline Larynx (D.mean) & $0.2 \pm 2.0$ & $1.0 \pm 2.1$ & $1.1 \pm 2.8$ & $0.9 \pm 1.7$ & 3.2 & 5.9 & 7.1 & 4.0 \\
\hline
\end{tabular}

Note: percentage (\%) was normalized to $60 \mathrm{~Gy}$ for OARs and to their corresponding prescription doses to target structures. RU: range uncertainties; SE: setup errors; SREs: combination of range uncertainties and setup errors; $\Delta \max (\%)$ presents the worst-case scenario: maximal reduction for target coverage and maximal dose increment for OARs.

and SREs for proton plans, and only SE for VMAT. The average variations of target coverage (CTV and PTV) caused by RU alone were smaller than those from SE alone for proton cases. The overall worst-case scenario coverage deviations were found to be comparable for proton (SREs) and VMAT (SE) plans: $-1.6 \% \pm 1.9 \%$ versus $-1.7 \% \pm 1.4 \%$ for CTV $54,-1.9 \% \pm 1.6 \%$ versus $-1.3 \% \pm$ $1.5 \%$ for CTV $60,-2.5 \% \pm 1.6 \%$ versus $-4.4 \% \pm 1.3 \%$ for PTV 54 and $-2.9 \% \pm$ $1.7 \%$ versus $-3.5 \% \pm 1.9 \%$ for PTV60. More than $5 \%$ drops in D98\% of CTV54 was found for 3 proton $(-6.0 \%,-6.8 \%$ and $-7.1 \%)$ and 1 photon patient $(-6.2 \%)$, and more than $5 \%$ decreases in D98\% of CTV60 was found for 3 proton $(-5.1 \%,-6.9 \%$ and $-7.3 \%)$ and 2 photon patients $(-6.1 \%$ and $-6.2 \%)$, which was also considered to be similar across the two modalities. No multiple instances of 5\% or more loss in CTV D98\% were found for any single patient.

While only dose variations from SE were evaluated for the VMAT plans, impacts of RU, SE and SREs on doses to OAR were reported separately for proton plans. The contralateral submandibular gland, located at the distal end of the proton beam and often bordering the target volume, was on average more sensitive to RU $(0.2 \% \pm 13.3 \%)$ than SE $(0.1 \% \pm 5.0 \%)$ for all 10 patients. Especially, when there was a direct overlap with target volume, the sensitivity of dose variations to RU increased because of its small volume. The SREs further increased the dose variations $(-0.1 \% \pm 13.0 \%)$. The same trend was observed for oral cavity, but with less magnitude due to the structure's larger total volume than submandibular gland. In contrast, the OARs located laterally to the proton beam, 
such as brainstem and cord, showed dominant sensitivity to SE, rather than RU. The dose variations caused by SE, RU and SREs were $0.3 \% \pm 2.7 \%, 0.6 \% \pm 9.6 \%$, $1.0 \% \pm 10 \%$ for brainstem and $0.1 \% \pm 1.0 \%, 2.2 \% \pm 2.2 \%, 2.2 \% \pm 2.6 \%$ for cord, respectively.

Figure 3 presents boxplots of robustness metrics under worst case scenarios for target coverage and dose to OARs. The legend indicates maximum, $75^{\text {th }}$ percentile, median, $25^{\text {th }}$ percentile and minimum values of each metric from top to bottom. Although the box values (between $75^{\text {th }}$ and $25^{\text {th }}$ percentile) of CTV $98 \%$ were lower for proton cases, overall range of variations were about the same, and the difference was $\sim 1 \%$. However, the box values of PTV D95\% were higher for proton cases. Larger variations on D95\%, especially for PTV54 were observed. While larger dose variations were found for brainstem and cord, safe dose to
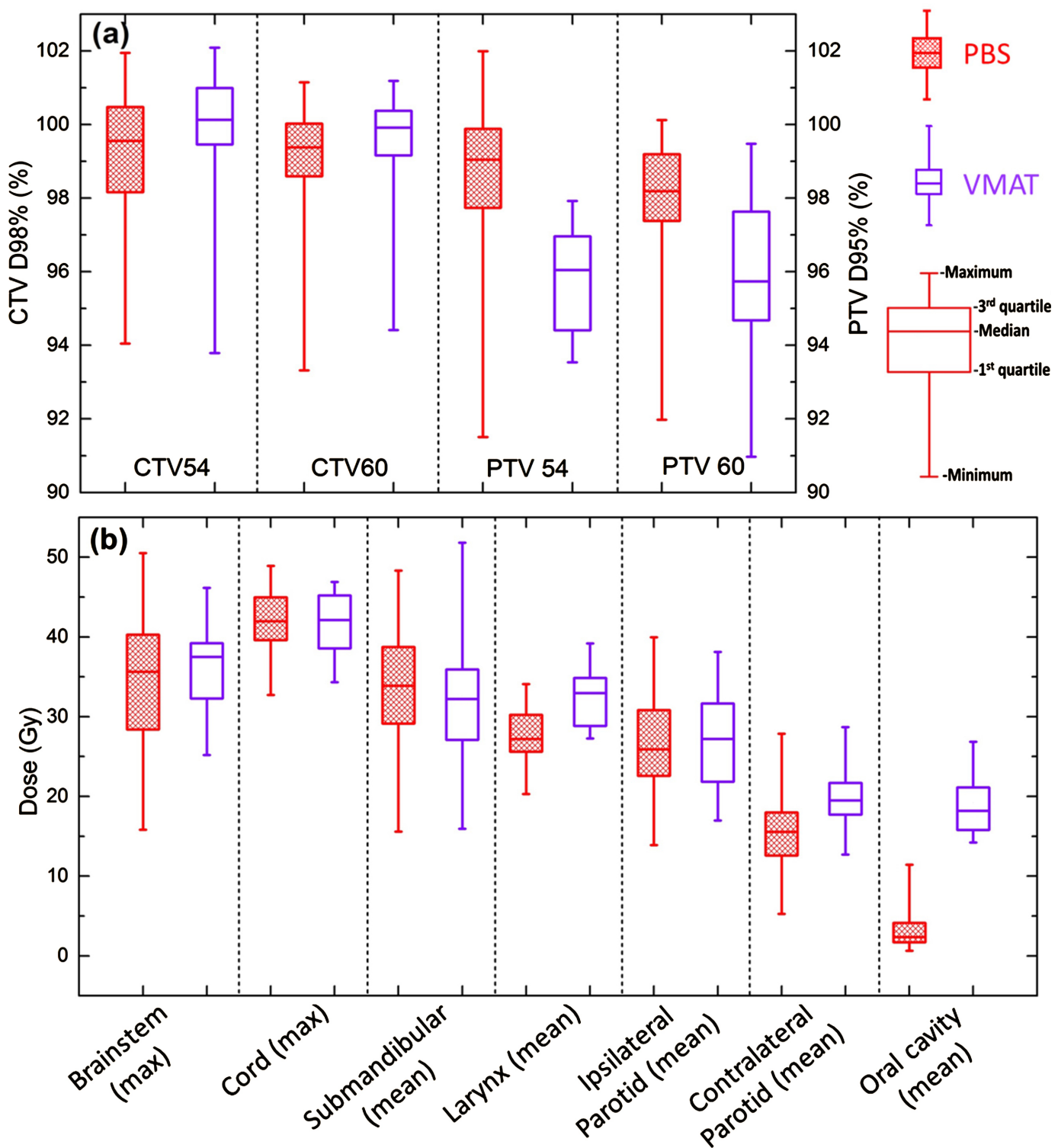

Figure 3. Comparison of dose variations on D98\% of CTV54, CTV60 (a) and OARs (b) for proton and VMAT plans in robustness study. 
cord and brainstem were generally maintained [16], as shown in Figure 3.

Table 3 presented the direct comparison of dosimetric metrics with and without considering the perturbations of RU and SE. In general, it showed very similar mean and deviation on dose metrics of CTV and PTV between PBS and VMAT. The mean doses to all OARs with perturbations were very similar to their mean planning dose while the deviation increased as expected. The dose to cord were $41.8 \pm 3.6$ Gy for PBS and $41.7 \pm 3.9$ Gy for VMAT with perturbations and the dose to brainstem were $35.2 \pm 8.4$ Gy for PBS and $36.2 \pm 5.1$ Gy for VMAT, which were also about the same range.

\section{Discussion}

This study presented a novel field geometry for $\mathrm{HN}$ patients receiving proton PBS treatment. The results of treatment planning comparisons between proton SFUD PBS delivery technique and VMAT photon radiotherapy for HN were reported, focusing on target coverage, OAR sparing and plan robustness for plans generated using commercial TPS (without robust optimization). The planning method containing two PBS posterior-oblique fields (POF) was first developed and used to treat $\mathrm{HN}$ patients at our institution. Considering the shape of typical HN target volumes, the selection of POF was used to improve the sparing of normal organs anterior to the target volumes, and reduce their treatment related toxicities. In contrast to VMAT plans, this goal could be achieved for most of the proton $\mathrm{HN}$ patients when there was no direct overlap between organs and target volumes. However, verification plans based on repeated CT scanning showed potentially large coverage variations to nodal volumes

Table 3. Mean and standard deviation of target and OAR dosimetric metrics with and without considering the perturbations of RU and SE in $10 \mathrm{HN}$ patients for proton and VMAT plans.

\begin{tabular}{ccccc}
\hline \multirow{2}{*}{ VOIs/Dose Metrics (Gy) } & \multicolumn{4}{c}{ Mean $\pm \Delta$ without perturbations } \\
\cline { 2 - 5 } & PBS & VMAT & PBS & vith perturbations \\
\hline PTV54 (D95\%) & $54.4 \pm 0.4$ & $54.1 \pm 0.4$ & $53.1 \pm 1.1$ & $51.7 \pm 0.8$ \\
PTV60 (D95\%) & $60.1 \pm 0.3$ & $59.9 \pm 0.3$ & $58.6 \pm 1.0$ & $57.7 \pm 1.2$ \\
CTV54 (D98\%) & $54.3 \pm 0.4$ & $54.9 \pm 0.6$ & $53.3 \pm 1.1$ & $53.9 \pm 0.9$ \\
CTV60 (D98\%) & $60.3 \pm 0.3$ & $60.6 \pm 0.4$ & $59.3 \pm 0.9$ & $59.7 \pm 1.0$ \\
Brainstem (D.max) & $35.7 \pm 7.2$ & $36.0 \pm 4.9$ & $35.2 \pm 8.4$ & $36.2 \pm 5.1$ \\
Cord (D.max) & $40.3 \pm 4.0$ & $39.8 \pm 3.7$ & $41.8 \pm 3.6$ & $41.7 \pm 3.9$ \\
Oral cavity (D.max) & $3.7 \pm 2.3$ & $18.6 \pm 3.1$ & $3.4 \pm 2.4$ & $18.7 \pm 3.6$ \\
Submandibular (D.mean) & $33.3 \pm 5.7$ & $32.2 \pm 7.9$ & $33.6 \pm 8.1$ & $32.8 \pm 8.5$ \\
Contralatral Parotid (D.mean) & $15.4 \pm 3.5$ & $19.5 \pm 2.7$ & $15.5 \pm 4.6$ & $19.7 \pm 3.6$ \\
Ipsilateral Parotid (D.mean) & $26.8 \pm 5.5$ & $26.8 \pm 4.7$ & $25.9 \pm 6.2$ & $27.2 \pm 6.0$ \\
Larynx (D.mean) & $27.2 \pm 2.7$ & $31.7 \pm 3.9$ & $27.7 \pm 3.1$ & $32.3 \pm 3.7$ \\
\hline
\end{tabular}

Note: $\Delta$ : Standard Deviations. 
located in the lower neck [17]. These nodal targets were typically shallow, from an anterior beam direction. Therefore, POF had to travel a longer beam path to reach them. Loose tissues on the back of the neck can fold differently from day to day and it was a challenge to achieve the goal of reproducibility for this loose tissue, especially for larger/older patients. Neither surface guidance with optical images, nor $\mathrm{kV}$ images during patient setup, are capable of flagging this change of tissue shape on the back of the patient's neck, which could significantly impact the POF proton beam path, and translate into range errors and reduced target coverage. However, it also showed consistency and robustness of the POF to target coverage and OAR sparing for volumes superior to the thyroid notch. Therefore, a revised planning method was proposed to separate the target volume into two sets, depending on their locations relative to the thyroid notch, and to add an anterior field to cover the lower target set [18]. Gradient dose matching was applied to create a "soft dose buffer" between two abutting field sets to reduce planning complications and dose uncertainties associated with the junction. The revised method has become our standard approach for proton treatment of HN patients.

One novelty of this work originates from the employment of gradient dose matching for the anterior and posterior field target sets in HN planning with a PBS technique. Different from the field matching in craniospinal irradiation, all fields here share the same isocenter in HN proton therapy and no couch shift is required between fields during treatment. Along with the fact that patient's head and neck area is immobilized by a thermoplastic mask, that effectively limits the patient's intrafractional movement along craniocaudal direction, so variations in daily setup should result in a wash-out effect along the junction [19], and large systematic setup errors are restricted for HN treatment through the use of accurate online imaging systems. According to the mathematic model for gradient field match developed by Lin [12], given a systematic setup error of $2 \mathrm{~mm}$, maximal $\sim 5 \%$ dose errors are expected at junction of $4 \mathrm{~cm}$ using gradient dose match. Thus, a junction length of $4 \mathrm{~cm}$ (shown in Figure 2) is considered to be reasonable to maintain a safe dose to critical organs, such as cord, and uniform coverage to target volumes in the junction area by adding the wash-out effect due to daily setup variations.

Another novelty comes from the selection of proton beam angles. Posterior fields provide sparing on the OARs anterior to treatment volumes, including optical system, as well as organs and tissues in oral cavity. However, the dose deviation caused by range uncertainties, which could be the dominant uncertainty factor as illustrated in this study, has to be assessed during plan evaluation. Large dose variations are generally expected for distally located small organs, such as the submandibular gland, especially when overlap with target volumes is present. In contrast to VMAT, higher mean dose is often observed for the submandibular glands overlapping with target volumes in a proton plan because extra margin is typically added distally to accommodate range uncertain- 
ties, so more normal tissue volume distal to the PTV receives the prescribed dose. However, Proton could provide significant sparing when no direct overlap occurs, such as for patient 6. Moreover, critical organs such as brainstem and spinal cord are placed laterally to the field to avoid unpredicted dose uncertainties caused by range uncertainty and enhanced radiobiological effect at the distal end of the Bragg peak [20]. It is easy to understand why setup uncertainties dominate the dose variations for these laterally positioned organs, as indicated in this study. Maximal cord dose between $45 \mathrm{~Gy}$ and $50 \mathrm{~Gy}$ was found for two patients ( 1 and 3 ) in a specific combination of SE and RU in their robustness study. Concerns on safe cord dose are reduced not only because minimal $(<0.5 \mathrm{cc})$ volume received dose of $45 \mathrm{~Gy}$ and above, these values represent the cord doses in the worst case scenario (consider SE as systematic errors through treatment course) and daily setup variation is ignored. Moreover, bony landmarks proximal to these organs, such as cervical vertebrae, are generally considered for patient setup to confirm the accuracy of patient alignments.

The robustness evaluation in this study is limited to SE and RU. The effect of patient's inter- and intra-fractional shifts, which could potentially smear the dose deviations predicated by the uncertainty study [21], were not investigated. With modern imaging guided therapy, the risk of $2.25 \mathrm{~mm} \mathrm{SE}$ in all three directions simultaneously is low, especially for $\mathrm{HN}$ cases with immobilization devices and special attention given to precise patient positioning. Further research is needed to investigate the dose uncertainties due to anatomic changes using online volumetric imaging [22] or repeating CT scans [23], as well as variation in relative biological effectiveness [24]. It would also be desirable to explore robust optimization algorithms during plan optimization to simultaneously strive for a robust plan with optimal OAR sparing [25] [26]. Furthermore, with the development of fast and accurate dose calculation algorithms [27] and advanced online imaging analysis tools [28], application of adaptive proton therapy on $\mathrm{HN}$ treatment should also be considered.

\section{Conclusion}

This study presented proton PBS treatments with a novel proton beam geometry of three SFUD fields for HN patients. The robustness of the proton plans was assessed based on RU and SE, and was compared to VMAT results. It demonstrated that a proton plan with three SFUD fields can lead to robustness similar to that of VMAT, even without robust optimization algorithms, while providing significant sparing to larynx, contralateral parotid and oral cavity.

\section{Acknowledgements}

We would like to thank the radiation oncology group in Roberts Proton Therapy Center at the University of Pennsylvania for their continuous supports on this project. This research did not receive any specific grant from funding agencies in the public, commercial, or not-for-profit sectors. 


\section{References}

[1] Huguenin, P.U., Taussky, D., Moe, K., Meister, A., Baumert, B., Lutolf, U.M., et al. (1999) Quality of Life in Patients Cured from a Carcinoma of The head and Neck by Radiotherapy: The Importance of the Target Volume. International Journal of Radiation Oncology • Biology • Physics, 45, 47-52. https://doi.org/10.1016/S0360-3016(99)00128-5

[2] Deasy, J.O., Moiseenko, L., Marks, L., Chao, J., Nam, J. and Eisbruch, A. (2010) Radiotherapy Dose-Volume Effects on Salivary Gland Function. International Journal of Radiation Oncology • Biology • Physics, 76, S58-S63.

https://doi.org/10.1016/j.ijrobp.2009.06.090

[3] Bar Ad, V., Lin, H.B., Hwang, W.T., Deville, C., Dutta, P.R., Tochner, Z., et al. (2012) Larynx-Sparing Techniques Using Intensity-Modulated Radiation Therapy for Oropharyngeal Cancer. Medical Dosimetry, 37, 383-386. https://doi.org/10.1016/j.meddos.2012.02.004

[4] Ahn, P.H., Lukens, J.N., Teo, B.K.K., Kirk, M. and Lin, A. (2014) The Use of Proton Therapy in the Treatment of Head and Neck Cancers. The Cancer Journal, 20, 421-426. https://doi.org/10.1097/PPO.0000000000000077

[5] Unkelbach, J., Bortfeld, T., Martin, B.C. and Soukup, M. (2009) Reducing the Sensitivity of IMPT Treatment Plans to Setup Errors and Range Uncertainties via Probabilistic Treatment Planning. Medical Physics, 36, 149-163. https://doi.org/10.1118/1.3021139

[6] Barten Danique, L.J., Tol Jim, P., Dahele, M., Slotman Ben, J. and Verbakel Wilko, F.A.R. (2016) Comparison of Organ-at-Risk Sparing and Plan Robustness for Spot-Scanning Proton Therapy and Volumetric Modulated Arc Photon Therapy in Head-and-Neck Cancer. Medical Physics, 42, 6589-6598.

https://doi.org/10.1118/1.4933245

[7] Malyapa, R., Lowe, M., Bolsi, A., Lomax, A.J., Weber, D.C. and Albertini, F. (2016) Evaluation of Robustness to Setup and Range Uncertainties for Head and Neck Patients Treated with Pencil Beam Scanning Proton Therapy. International Journal of Radiation Oncology • Biology• Physics, 95, 154-162. https://doi.org/10.1016/j.ijrobp.2016.02.016

[8] Yu, J., Zhang, X.D., Liao, L., Li, H., Zhu, R., Park, P.C., et al. (2016) Motion-Robust Intensity-Modulated Proton Therapy for Distal Esophageal Cancer. Medical Physics, 43, 1111-1118. https://doi.org/10.1118/1.4940789

[9] Liu, W. and Mohan, R. (2012) Robustness Evaluation and Robust Optimization of IMPT Plans Based on Per-Voxel Standard Deviation of Dose Distributions. Medical Physics, 39, 3850. https://doi.org/10.1118/1.4735715

[10] van der Voort, S., van de Water, S., Perko, Z., Heijmen, B., Lathouwers, D. and Hoogeman, M. (2016) Robustness Recipes for Minimax Robust Optimization in Intensity Modulated Proton Therapy for Oropharyngeal Cancer Patients. International Journal of Radiation Oncology • Biology• Physics, 95, 163-170. https://doi.org/10.1016/j.ijrobp.2016.02.035

[11] van de Water, S., van Dam, I., Schaart, D.R., Al-Mamgani, A., Heijmen, B.J. and Hoogeman, M.S. (2016) The Price of Robustness; Impact of Worst-Case Optimization on Organ-at-Risk Dose and Complication Probability in Intensity-Modulated Proton Therapy for Oropharyngeal Cancer Patients. Radiotherapy and Oncology, 120, 56-62.

https://doi.org/10.1016/j.radonc.2016.04.038

[12] Lin, H.B., Ding, X.F., Kirk, M., Liu, H.Y., Zhai, H.F., Hill-Kayser, C.E., et al. (2014) 
Supine Craniospinal Irradiation Using a Proton Pencil Beam Scanning Technique Without Match Line Changes for Field Junctions. International Journal of Radiation Oncology • Biology • Physics, 90, 71-78. https://doi.org/10.1016/j.ijrobp.2014.05.029

[13] Lin, H., Shen, J., McDonough, J., Tochner, Z. and Both, S. (2015) Should the Machine-Related Range Shifter (MRS) Be Replaced by a Patient-Related Range Shifter (PRS) to Preserve the Scanning Beam Dosimetric Advantages? Medical Physics, 42 , 3495-3496. https://doi.org/10.1118/1.4925055

[14] Both, S., Shen, J., Kirk, M., Lin, L., Tang, S., Alonso-Basanta, M., et al. (2014) Development and Clinical Implementation of a Universal Bolus to Maintain Spot Size during Delivery of Base of Skull Pencil Beam Scanning Proton Therapy. International Journal of Radiation Oncology • Biology • Physics, 90, 79-84.

https://doi.org/10.1016/j.ijrobp.2014.05.005

[15] Lowe, M., Albertini, F., Aitkenhead, A., Lomax, A.J. and MacKay, R.I. (2016) Incorporating the Effect of Fractionation in the Evaluation of Proton Plan Robustness to Setup Errors. Physics in Medicine \& Biology, 61, 413-429.

https://doi.org/10.1088/0031-9155/61/1/413

[16] Taheri-Kadkhoda, Z., Bjork-Eriksson, T., Nill, S., Wilkens, J.J., Oelfke, U., Johansson, K.A., et al. (2008) Intensity-Modulated Radiotherapy of Nasopharyngeal Carcinoma: A Comparative Treatment Planning Study of Photons and Protons. Radiation Oncology, 3, 4. https://doi.org/10.1186/1748-717X-3-4

[17] Liang, X., Lin, A., Tang, S., Zhai, H., Kirk, M., Kalbasi, A., et al. (2014) Proton PBS Plan Design and Robustness Evaluation for Head and Neck Cancer. International Journal of Radiation Oncology • Biology • Physics, 90, S944.

[18] Apinorasethkul, O.K.M., Teo, K., Swisher-McClure, S., Lukens, J.N. and Lin, A. (2017) Pencil Beam Scanning Proton Therapy vs. Rotational Arc Radiation Therapy: A Treatment Planning Comparison for Postoperative Oropharyngeal Cancer. Medical Dosimetry, 42, 7-11. https://doi.org/10.1016/j.meddos.2016.09.004

[19] van Kranen, S., van Beek, S., Rasch, C., van Herk, M. and Sonke, J.J. (2009) Setup Uncertainties of Anatomical Sub-Regions in Head-and-Neck Cancer Patients after Offline Cbct Guidance. International Journal of Radiation Oncology • Biology • Physics, 73, 1566-1573. https://doi.org/10.1016/j.ijrobp.2008.11.035

[20] Matsumoto, Y., Matsuura, T., Wada, M., Egashira, Y., Nishio, T. and Furusawa, Y. (2014) Enhanced Radiobiological Effects at the Distal End of a Clinical Proton Beam: In Vitro Study. Journal of Radiation Research, 55, 816-822.

https://doi.org/10.1093/jrr/rrt230

[21] Lin, L., Souris, K., Kang, M., Glick, A., Lin, H., Huang, S., et al. (2017) Evaluation of Motion Mitigation Using Abdominal Compression in the Clinical Implementation of Pencil Beam Scanning Proton Therapy of Liver Tumors. Medical Physics, 44, 703-712. https://doi.org/10.1002/mp.12040

[22] Kurz, C., Nijhuis, R., Reiner, M., Ganswindt, U., Thieke, C., Belka, C., et al. (2016) Feasibility of Automated Proton Therapy Plan Adaptation for Head and Neck Tumors Using Cone Beam CT Images. Radiation Oncology, 11, 64. https://doi.org/10.1186/s13014-016-0641-7

[23] Li, H., Zhang, X., Park, P., Liu, W., Chang, J., Liao, Z., et al. (2015) Robust Optimization in Intensity-Modulated Proton Therapy to Account for Anatomy Changes in Lung Cancer Patients. Radiotherapy and Oncology, 114, 367-372. https://doi.org/10.1016/j.radonc.2015.01.017

[24] Tseung, H.S.W.C., Ma, J.S., Kreofsky, C.R., Ma, D.J. and Beltran, C. (2016) Clini- 
cally Applicable Monte Carlo-Based Biological Dose Optimization for the Treatment of Head and Neck Cancers with Spot-Scanning Proton Therapy. International Journal of Radiation Oncology • Biology • Physics, 95, 1535-1543.

https://doi.org/10.1016/j.ijrobp.2016.03.041

[25] Van der Voort, S., van de Water, S., Perko, Z., Heijmen, B., Lathouwers, D. and Hoogeman, M. (2016) Robustness Recipes for Minimax Robust Optimization in Intensity Modulated Proton Therapy for Oropharyngeal Cancer Patients. International Journal of Radiation Oncology • Biology• Physics, 95, 163-170.

https://doi.org/10.1016/j.ijrobp.2016.02.035

[26] Stützer, K., Lin, A., Kirk, M. and Lin, L. (2017) Superiority in Robustness of Multifield Optimization over Single-Field Optimization for Pencil-Beam Proton Therapy for Oropharynx Carcinoma: An Enhanced Robustness Analysis. International Journal of Radiation Oncology • Biology • Physics, 99, 738-749. https://doi.org/10.1016/j.ijrobp.2017.06.017

[27] Souris, K., Lee, J.A. and Sterpin, E. (2016) Fast Multipurpose Monte Carlo Simulation for Proton Therapy Using Multi- and Many-Core CPU Architectures. Medical Physics, 43, 1700-1712. https://doi.org/10.1118/1.4943377

[28] Wang, P., Yin, L.S., Zhang, Y.W., Kirk, M., Song, G., Ahn, P.H., et al. (2016) Quantitative Assessment of Anatomical Change Using a Virtual Proton Depth Radiograph for Adaptive Head and Neck Proton Therapy. Journal of Applied Clinical Medical Physics, 17, 427-440. https://doi.org/10.1120/jacmp.v17i2.5819 